\title{
Design and Methods of a Randomized Cross-over Clinical Trial of Unfiltered Cigarettes on Smoking Behavior and Toxicant Exposure: Study Protocol for a Randomized Cross-over Clinical Trial
}

Eyal Oren ( $\nabla$ eoren@sdsu.edu )

San Diego State University

Kim Pulvers

California State University San Marcos

Devan R. Romero

California State University, San Marcos

Casey Barber

San Diego State University

Erika Carter

California State University, San Marcos

Thomas E. Novotny

San Diego State University

\section{Study Protocol}

Keywords: nicotine, tobacco, topography, exposure, policy, environmental

Posted Date: March 17th, 2020

DOl: https://doi.org/10.21203/rs.3.rs-17488/v1

License: (1) (1) This work is licensed under a Creative Commons Attribution 4.0 International License. Read Full License 


\section{Abstract}

Background: Plastic filters on cigarette butts are a widespread source of non-biodegradable, toxic environmental waste. State and local legislation to ban the sale of single-use cigarettes may be considered to prevent this waste, but scientific evidence on the impact of switching smokers to unfiltered cigarettes on smoking behavior and toxicant exposures is needed to inform this policy. We have designed an open-label, randomized, 9-week, cross-over clinical trial of adult filtered cigarette smokers who switch to unfiltered cigarettes.

Methods: The trial involves a 1-week baseline period, 2 weeks of smoking filtered or unfiltered cigarettes (groups randomly assignd), a 3-week washout period, another 1-week baseline period, and a crossover to 2weeks of smoking the opposite condition (filtered or unfiltered cigarettes). We will determine changes in: 1) observed topography (i.e., puff count, puff volume) and cigarettes smoked per day (via butt counts and selfreport); 2) expired carbon monoxide (CO), urinary cotinine, 4-(methylnitrosamino)-1-(3-pyridyl)-1-butanol (NNAL), and volatile organic compound (VOC) excretion; and 3) knowledge and attitudes toward unfiltered cigarettes, satisfaction with smoking, and intention to quit if they were not able to smoke filtered cigarettes.

Discussion: This comprehensive proof-of-principle study will provide preliminary evidence to develop a research project with a larger sample size. Study results will inform proposed state or local legislation to ban the sale of single-use filtered cigarettes in order to eliminate the environmental impact of cigarette butt waste stemming from the single-use plastic filter.

Trial Registration: NCT03749876

\section{Introduction}

\section{Background and rationale $\{6 \mathrm{a}\}$}

Cellulose acetate (plastic) filters on cigarette butts are a widespread source of non-biodegradable environmental waste that may be toxic to marine organisms, aquatic environments, and possibly to human and animal health (1-4). Cigarette butts are, in fact, the main littered item found during beach and urban cleanups each year throughout the world and adversely affect storm water drainage, beaches, neighborhoods, and other natural environments. There is a widespread perception among smokers and non-smokers that filters provide a "safer" cigarette (5). Most smokers and non-smokers do not know that the filters attached to more than $99 \%$ of cigarettes sold in the United States are made of non-biodegradable plastic. However, cigarette filters have also been deemed 'unproved in reducing harms to the average smoker' by the US Surgeon General and the National Cancer Institute $(6,7)$. Data comparing the relative risks of smoking among age-matched cohorts of smokers across 50 years show that overall mortality, as well as the smoking-attributable risks for morbidity and mortality from lung cancer, heart disease, and COPD, increased over the time that filters have been standardized attachments to cigarettes $(8,9)$. State and local governments have jurisdiction to ban the sale of various tobacco products, and in 2014 , a committee of the California Assembly considered, for environmental reasons, a bill to ban the sale of singleuse filtered cigarettes (10). In 2019, a bill to ban the sale of filtered cigarettes as well as plastic tips for 
cigars, electronic cigarettes, and other tobacco waste products was passed by the California Senate and is pending in the Assembly (11). Additional scientific evidence on the human consequences of removing single-use filters from cigarettes is needed to understand what, if any, health and behavioral consequences may result from an environmental policy eliminating cellulose acetate-filtered cigarettes from the tobacco market.

\section{Study Objectives $\{7\}$}

The overall study research question is: What is the impact of switching smokers of filtered cigarettes to unfiltered cigarettes on smoking behavior and toxic exposures? The Specific Aims of the study are as follows: 1) Determine smokers' satisfaction, and attitudes towards smoking cigarettes if they were to switch from smoking filtered to unfiltered cigarettes. 2) Measure changes in smoking topography (i.e., puff count, puff volume) and cigarettes smoked per day (via butt counts and self-report) among smokers who change to unfiltered cigarettes for two weeks compared with these measures while smoking filtered cigarettes. 3) Measure changes in urinary cotinine, 4-(methylnitrosamino)-1-(3-pyridyl)-1-butanol (NNAL), and volatile organic compound (VOC) excretion among smokers who smoke unfiltered cigarettes for two weeks compared to smoking filtered cigarettes. Study results will inform proposed state or local legislation to ban the sale of single-use filtered cigarettes in order to eliminate the environmental impact of cigarette butt waste stemming from the plastic filter.

\section{Overview and Trial Design $\{8\}$}

We will conduct an open-label, randomized, 9-week, two-sequence, two-treatment, crossover clinical trial of 40 adult filtered cigarette smokers who switch to unfiltered cigarettes. There will be a 1-week baseline period, 2-weeks of smoking filtered or unfiltered cigarettes (groups randomly determined), a 3-week washout period, another 1-week baseline period, and a crossover to 2-weeks of smoking the opposite condition (filtered or unfiltered cigarettes). This proof-of-principle study will provide preliminary evidence to develop a follow-up research project with a larger sample size.

We will use validated computer-administered surveys from baseline, intervention, and post-intervention time-points to assess changes in knowledge and attitudes regarding smoking of filtered and unfiltered cigarettes. We will use a computerized device (CReSS Pocket) to measure smoking topography over 5 daily eight-hour periods per week; the device is taken home and readings are recorded in naturalistic settings. This device permits convenient recording of smoking topography throughout the day, with time- and datestamped data, allowing us to know when and how the cigarettes are smoked and thus whether the pattern of smoking has changed. Participants will collect and return cigarette butts each week and self-report the number of cigarettes smoked per day. We will assess exhaled carbon monoxide (CO) using a breath monitor each week; we will also collect urine samples during baseline, intervention, and washout periods to measure cotinine, NNAL, and VOC excretion (normalized with urinary creatinine).

\section{Methods: Participants, Interventions And Outcomes}

\section{Study setting $\{9\}$}


The study takes place in San Diego, California, United States, with participants screened remotely by Institution A and then physically attending a smoking laboratory at Institution B. All laboratory and other samples are measured and analyzed in-house.

\section{Eligibility criteria $\{10\}$}

Inclusion criteria. Participants must meet all of the following criteria: age 21-65 years; smokers of $\geq 5$ cigarettes per day (cpd) on $\geq 25$ days per month for $\geq$ one year; smokers of Camel or Pall Mall filtered cigarettes for at least two weeks prior to enrollment, and willing to continue using this brand during the study; fluent in English; have regular access to a telephone; and have transportation to attend all scheduled visits. Participants must primarily ( $\geq 50 \%$ of the time) smoke cigarettes, allowing for secondary use of other tobacco products. Furthermore, we will verify their regular smoking status upon enrollment with a measured expired air CO level of $\geq 10 \mathrm{ppm}$ (12).

Exclusion criteria. Participants will be excluded if they are currently in a smoking cessation program or another clinical trial; are using nicotine replacement therapy or medication which aids smoking cessation in the past month, including Zyban (bupropion), Catapres (clonidine), Pamelor (nortriptyline), or Chantix (varenicline); or have tried to quit or cut down on smoking cigarettes in the past month. Women who are pregnant, breastfeeding, or planning to become pregnant in the next six months will be excluded. Medical exclusion criteria include (1) self-reported uncontrolled diabetes mellitus, presence of any cardiovascular issue in the past 30 days including heart attack, stroke, severe angina (i.e., chest pain), hypertension, ischemic heart disease, vascular disease, or any other cardiovascular disease, (2) presentation to the enrollment visit with a systolic blood pressure greater than $160 \mathrm{~mm} \mathrm{Hg}$ or a diastolic blood pressure greater than $105 \mathrm{~mm} \mathrm{Hg}$ (verified by taking two blood pressure readings), (3) self-reported hospitalization for psychiatric issues, (4) being mentally or physically unfit to participate in the study, or (5) current dependence on a substance other than nicotine.

\section{Who will take informed consent? $\{26 \mathrm{a}\}$}

Informed consent will be assured during the smoking laboratory visit by trained research assistants. All consent will be recorded in person. Participants will be informed during the consent process that they may withdraw from the study at any time for any reason. Participants will also be provided the purpose of the study, study objectives, as well as how study success will be measured.

\section{Interventions}

\section{Intervention description $\{11 \mathrm{a}\}$}

The first week will provide a baseline measurement of smoking behavior and urinary measurements. The next 2 weeks (Weeks 2 and 3) will involve filtered or unfiltered cigarette smoking, followed by a 3-week washout period (Weeks 4-6). Week 7's visit will initiate a post-washout, 1-week baseline period. The crossover condition will be implemented for Weeks 8-9. Study cigarettes will be provided during the two switching trial periods (4 weeks total). Topography will be measured on 5 days of smoking during the two 
baseline weeks (enrollment and post-washout) and during the four weeks of trial; expired $\mathrm{CO}$, weight, and survey measures will be assessed at all visits.

Study cigarettes $\{6 \mathrm{~b}\}$. Two brands of cigarettes are currently available in a filtered and unfiltered design: Camel and Pall Mall. Participants will be provided supplies of one of these two brands throughout the study period based on their preference. After baseline measurements at week 2, they will be randomly assigned to smoke two weeks of filtered or two weeks of unfiltered study cigarettes. The number provided will be the average cpd smoked for each previous week plus 10\%. For example, a participant reporting smoking $10 \mathrm{cpd}$ in the previous week would be provided with a supply of cigarettes equaling $11 \mathrm{cpd}$ (10 cpd plus 10\%).

Encouraging adherence $\{11 \mathrm{c}\}$. To support participants' adherence to the interventions, study staff will review relevant expectations in detail for participants at each laboratory visit. A reminder card with the week's instructions and next laboratory appointment are provided as well as a troublesthooting guide for operation of the CReSS Pocket topography device.

\section{Outcomes $\{12\}$}

\section{Knowledge and attitudes toward unfiltered cigarettes}

Participants will be asked at enrollment and the final visit to what extent they believe that the filter on their brand of cigarettes (a) protects them from health problems caused by smoking and (b) makes smoking more pleasurable. Response options will include not at all, a little, quite a bit, and a great deal. In addition, they will be asked "If filtered cigarettes were no longer available, what if anything, would you change about your smoking patterns?" Response options will include increase number of cigarettes I smoke, nothing, cut back, and try to quit smoking altogether. Participants will also be asked the purpose of putting filters on cigarettes, with the following response options (more than one answer accepted): making cigarettes safer to smoke, making it easier to begin smoking, making it more pleasurable to smoke, selling more cigarettes, making cigarettes cheaper, and other. Finally, participants will be asked what filters are made of with the following options (more than one answer accepted): cotton, food starch, asbestos, plastic/cellulose acetate, and other.

Qualitative questions at enrollment and the final visit will include: 1) Almost all cigarettes smoked in the United States have a filter; most people have dropped their cigarette butts (with the filter) somewhere outdoors at some time, and so do you think that these discarded butts are a problem for the environment? If so, why? If not, why not?, 2) What do you think should be done to provide the cigarette butts (and the attached filters) from adversely affecting the environment?, 3) Have you ever tried smoking cigarettes without filters? If yes, please briefly describe your experience... bad, good, or whatever; and 4) If filtered cigarettes were no longer available for purchase, what would likely be your response?

\section{Satisfaction with smoking}

At each visit, participants will smoke a cigarette in the lab and answer the following questions from the Cigarette Evaluation Scale (13) immediately after smoking: Was it satisfying?, Did it taste good?, Did it 
make you dizzy?, Did it calm you down?, Did it help you concentrate?, Did it make you feel more awake?, Did it reduce your hunger for food?, Did it make you feel nauseous?, Did it make you feel less irritable? Did you enjoy the sensations of the smoke in your throat and chest?, and Did it immediately reduce your craving for cigarettes? Each item is rated on a Likert scale ranging from 1, not at all to 7, extremely.

\section{Intention to quit}

Participants will be asked at each visit "What best describes your intentions to stop smoking completely, not even a puff?" Response options include: never expect to quit, may quit in the future but not in the next 6 months, will quit in the next 6 months, and will quit in the next 30 days (14). In addition, they will be asked whether they currently set a limit for how many cigarettes they smoke a day to decrease health risks from smoking (14).

\section{Nicotine dependence}

The Fagerström Test for Nicotine Dependence (15) will be administered at enrollment and the final visit, and a single item index of addiction to cigarettes (0-100) (16) will be established at every visit. The Brief Wisconsin Inventory of Smoking Dependence Motives will also be assessed at the Enrollment/Baseline and End visits (17).

\section{Cigarettes per day (self-report and butt count)}

Standard questions will determine how many days in the past month (Enrollment/Baseline visit), past week (weekly visits) and past two months (final visit) participants have smoked, as well as how many cpd they have smoked on those days. In addition, participants will return their cigarette butts in a sandwich-size Ziploc bag or glass jar each week to provide a validation of their self-report. These butts will be disposed of as toxic waste in approved containers. Previous studies have included butt counts with reliability set at $75 \%$ of returns (18). We will exclude butt count data from participants who do not return $75 \%$ of butts, and will provide incentive payments for returning cigarette butts.

\section{Smoking topography}

Behavioral adaptations are measured by smoking topography, including puff number/cigarette, average duration (sec), average inter-puff interval $(\mathrm{sec})$, average flow rate $(\mathrm{mL} / \mathrm{s})$, and average and total volume $(\mathrm{mL})$ (19).We will use a portable version of the Clinical Research Support System (a.k.a. CReSS Pocket) (Borgwaldt KC, North Chesterfield, VA) to measure topography over 5 ly daieight-hour periods per week: at baseline (Week 1), during the initial switch (Weeks 2-3), at post-washout baseline (Week 7), and again after the cross-over switch (Week 8-9). This computerized device permits convenient recording of cigarettes smoked throughout the day in the smokers' natural environment. Data are time- and date-stamped, allowing us to know when and how the cigarettes are smoked and thus whether the pattern of smoking has changed. Subjects will be trained in use of the device by the study team at the initial lab visit in a specialized facility designed with proper ventilation to accommodate indoor smoking research. Multiple days of measurement allow for assessment of reliability and sensitivity of topography changes as a 
function of the filter manipulation. Topographic measures by CReSS have been compared to direct observation via video recordings, and the CReSS has been demonstrated to be a valid measurement device (20).

\section{Carbon monoxide}

Expired air $\mathrm{CO}$ provides a measure of exposure to tobacco smoke and other fumes. A breath test will be conducted at each visit with the Micro + (Bedfont, Haddonfield, $\mathrm{NJ}$ ) to assess expired CO levels.

\section{Cotinine}

Cotinine is the main proximate metabolite of nicotine. Cotinine will be measured by liquid chromatographymass spectrometry (LCMS) (21) and normalized for creatinine from urine samples taken at each visit.

\section{Tobacco-specific nitrosamines}

Tobacco-specific nitrosamines (TSNAs) are carcinogens which are found in tobacco and tobacco smoke. Excretion (normalized for creatinine) of NNAL, a carcinogen biomarker will be measured by LCMS (21) from urine samples. In addition, carcinogenic VOCs, excreted as mercapturic acids, will be measured from urine samples. Both are useful biomarkers of changes in exposure to tobacco smoke $(22,23)$. These metabolites, along with expired $\mathrm{CO}$, provide a biomarker of exposure, thus creating a battery that reflects risks for smoking-induced diseases (24).

\section{Safety monitoring questions}

Respiratory effects will be assessed at every visit by asking about the presence of shortness of breath episodes or awakening from breathing difficulties during the past two weeks (yes/no). Nicotine toxicity will be assessed at every visit by asking whether the following symptoms have been experienced in the last two weeks: nausea and/or vomiting, nervous irritability beyond normal day to day stresses, tremors, rapid heart rate, nightmares, and chest pains (yes/no). Blood pressure and CO levels will also be monitored at every visit.

\section{Criteria for discontinuing or modifying allocated interventions $\{11 \mathrm{~b}\}$}

Serious adverse events will result in discontinuation of the intervention. These are defined as events that:

- have resulted in death;

- are life threatening;

- require inpatient hospitalization;

- result in persistent or significant disability/incapacity;

- result in a congenital anomaly/birth defect; or

- any other adverse event that, based upon appropriate medical judgment, may jeopardize the subject's health and may require medical or surgical intervention to prevent one of the other outcomes listed in this definition 
Study personnel will notify the Principal Investigator of any serious adverse events immediately after first awareness of the problem. The Principal Investigator will report serious adverse events to the IRB immediately.

\section{Participant timeline $\{13\}$}

There will be a 1-week baseline period, 2 weeks of smoking filtered or unfiltered cigarettes (determined at time of randomization), and a 3-week washout period, followed by post-washout baseline week, and a crossover to 2 weeks of smoking the opposite condition (Figure 1).

\section{Sample size $\{14\}$}

The study sample size is 40 participants, sufficient to detect moderate within-subject effect sizes $(f=0.35)$ with a moderate correlation between repeated measures ( $r>0.50)$. This $2 x 2$ crossover design has statistical power of $80 \%$ for a sample size of 20 for each group to detect an effect size of $10 \%$ change in average number of cigarettes smoked per day. We will try to recruit at least 10/40 non-White ethnicities. If subjects drop out in mid-trial, we will recruit replacements from the eligible pool or advertise for new subjects. We will recruit 20 women out of the 40 participants and randomize by sex stratum. Sex stratification is of interest both because men and women may metabolize smoke differently, and due to differences in cigarette preference and behavior.

\section{Recruitment $\{15\}$}

Recruitment will be accomplished using a combination of Craigslist, newspaper ads, and paper flyers, which are approaches that have been successfully used in previous studies by the study team $(25,26)$. Prospective participants will respond to advertisements via telephone or email to learn more about the study and to coordinate a time for phone screening to determine eligibility. Former participants in studies conducted at Institution B who have expressed interest in being contacted for future studies will be contacted and invited to complete the screening questionnaire.

\section{Assignment of interventions: Allocation $\{16 a, 16 b$, and $16 c\}$}

Once a participant is deemed eligible for the trial remotely by Institution A, trial personnel at Institution B will follow-up with the participant to schedule the initial trial appointment (Enrollment/Baseline Visit).

Participants who do not meet criteria will not be included in the trial. Recruitment and enrollment will be a continuous process (up to 10 participants at a time) which will enable us to utilize topography devices and lab facilities most effectively. Randomization, stratified by sex, is based on a table of random numbers and placed in sealed envelopes, with 20 assigned to be first switchers following their Enrollment/Baseline Visit at Institution B.

These participants will then be tested on individualized schedules (not as a group), with each participant committing to 9 weeks of trial time, including a washout period in mid-trial. First, all subjects will smoke 
filtered Camel or Pall Mall cigarettes for at least two weeks prior to beginning the trial (inclusion criteria). This will standardize their exposure to a brand that is also commonly available as an unfiltered variety.

\section{Assignment of interventions: Blinding}

\section{Blinding \{17a\}}

Investigators will be masked to group allocation, but participants and research staff will not, given their active study roles. DSMB members will be unblended to group allocation if necessary.

\section{Data collection and management}

\section{Plans for assessment and collection of outcomes $\{18 \mathrm{a}$ and 33$\}$}

There will be a total of eight lab visits scheduled, each with an incentive for attendance. As shown in Table 1 , these visits will occur weekly at baseline and during the first two weeks of cigarette use (Weeks 1-3), and also during the post-washout baseline and second two weeks of cigarette use (Weeks 7-9). During all visits, participants will provide breath samples to assess $\mathrm{CO}$, blood pressure and weight measurements, and will complete survey measures (see Table 1). At Weeks 1, 3, 7, and 9 participants will provide a urine sample to measure cotinine and carcinogen levels. All collected urine samples will be stored in a sub-eighty-degree freezer. Specimens will be shipped transported Institution B to Institution A for analysis of cotinine, creatinine, NNAL, and VOCs. Other specimens will be stored on the study site for analysis at a later time should additional funding become available.

During Visits 2-4 and 6-8, participants will arrive at the laboratory with their topography device, cigarette butts, and unused cigarettes (Weeks 3, 4, $7 \&$ 8). Data from the topography device will be downloaded so that the number of cigarettes logged can be compared to the total number of cigarettes/butts returned, as well as the number self-reported. Those deemed compliant with study procedures will be paid for their time and supplied with another weeks' supply of condition-assigned cigarettes. During visit 8 , the same procedure will be followed except it will be the final visit and no additional study cigarettes will be provided.

\section{Plans to promote participant retention and complete follow-up $\{18 \mathrm{~b}\}$}

Adherence to using the topography device will be incentivized by paying participants \$10/day for each day up to 5 days, which is checked at each lab visit. Compensation will be provided for collecting and returning cigarette butts $\$ 30$ a week for each week. These payments, combined with payment for attending the laboratory visit (\$20), totals up to $\$ 100$ for visits that occur during nine study weeks. For the post-washout baseline the compensation will be $\$ 75$. This reimbursement schedule will be reviewed with the participant at each visit (see Table 2). In addition, weekly visit reminder cards will be sent home with participants, which outline reminders of next appointment and visit expectations (collect all butts and use device 5 days).

\section{Data management $\{19\}$}


Study data will be collected and managed using REDCap, electronic data capture tools that are hosted through Institution A. REDCap is a secure, web-based application that is designed to support data capture for research studies. All of the data collected will be anonymized and password protected. Data will be entered shortly after collection. All REDCap data will be stored securely on a server at Institution A.

\section{Confidentiality \{27\}}

Confidentiality of data will be assured by assigning code numbers to survey data. Participants' identities will be in no way linked to their responses. Any paper documentation will be kept in a locked filing cabinet located at the study office. Data will only be accessible to personnel involved with this research. Access is limited by the research facility being locked at all times.

All information will be maintained on secured computers and a password protected laptop. The files on the laptop will be password-protected for added security. All electronic records will only contain de-identified information and all paper records, will be kept in an appropriate locked cabinet. The campus data network is protected by a perimeter firewall and the network within the campus network is further protected by another firewall.

\section{Statistical methods}

\section{Statistical methods for primary and secondary outcomes $\{20 \mathrm{a}\}$}

Descriptive statistics will be performed on baselines data to assess for any imbalances between groups in baseline demographics and pre-randomization characteristics. Stratified analyses and analyses of covariance will be performed to control for any baseline imbalances. To account for the cross-over design and repeated measures, we will measure changes in means of continuous measures using a mixed-effects model with fixed effects for period and treatment. The model variance will be fit using a compound symmetry correlation structure with default degrees of freedom, which assumes constant variance across periods. For testing hypotheses, paired and independent t-tests will be performed for within group and between group comparisons. All analyses will be performed using SAS 9.4 (27).

\section{Missing data $\{20 \mathrm{c}\}$}

Missing data will be minimized or avoided through extensive training of clinical research staff and repeated efforts to contact trial participants to obtain protocol-specified data. In the event of missing data, sensitivity analyses will be performed including a completer analysis and multiple imputation approaches.

\section{Oversight and monitoring}

\section{Safety monitoring plan and adverse event reporting and harms \{11b, 21a, 21b, and 22\}}

This study will be registered at ClinicalTrials.gov. A Data Safety and Monitoring Board (DSMB) consisting of a physician, a clinical pharmacologist, and a health behavioral scientist will be appointed from Faculty or collaborating institutions with relevant expertise who are not involved in this study. Data on serious adverse 
events, including severity, outcome, and management, will be included on individual participant files and in aggregate by treatment group and will be reviewed weekly. These data will be provided to the DSMB in a detailed report by an Institution A research assistant. Potential problems reportable to the DSMB include self-reported health issues such as respiratory problems, addiction or dependence, or nicotine toxicity as measured by carbon monoxide, blood pressure, or urinary biomarker levels. In the event of concerns, the committee will notify the PI to intervene and the medical consultant will be promptly consulted. The PI will assess the potential risks to the participant regarding continuation of the trial and report back to the committee on findings; the committee will then decide if the trial should continue or if the participant should be excluded.

Although smokers will be switching to unfiltered cigarettes for this trial, a two-week trial period is highly unlikely to provide any differential short or long-term risk to participants who smoke unfiltered cigarettes. The targeted cigarette brands are commercially available to the population already, and hence do not constitute a new product test. In addition, a physician-member of the research team will be on call to answer queries from the field staff regarding problems or questions from participants regarding switching to unfiltered cigarettes. A DSMB charter will be established outlining the committee members' responsibilities, meeting structure and deliverables, timeline and membership requirements, a written schedule of their individual activities, phone numbers, and copies of their informed consent forms.

\section{Auditing \{23\}}

All research staff have received human subjects research training and will receive appropriate training in recruitment, data collection, and management. Ongoing study progress will be tracked with regular monitoring by the PI with study and clinic personnel, training of all staff involved in the process, monthly research team meetings, and regular review to ensure that study targets are being met.

\section{Protections against risk}

We will be measuring biomarkers of carcinogen and nicotine exposures in order to assess the potential for long-term risk differences for unfiltered cigarette smokers vs. filtered cigarette smokers. In addition, because secondhand smoke is a combination of expired and side stream smoke, this exposure should be unaffected by the presence or absence of a filter.

We will be recruiting only current smokers, all of whom will have been warned about the health consequences of smoking in the informed consent procedures. We will train study staff to be nonjudgmental towards smokers, to reassure them of anonymity in reporting findings, to reinforce the scientific value of this study, and to provide feedback after completion of the study.

\section{Protocol amendment procedures}

Any modification(s) or amendment(s) to the study protocol will be reviewed as a study team, discussed with the DSMB, and ultimately shared for approval by the Institutional Review Board at Institution A prior to implementation. 
Dissemination plans $\{31$ a\}This project is expected to result in a number of oral presentations geared to specific local and national stakeholders. In addition, results of this study will be published and presented at Society for Research on Nicotine and Tobacco, American Public Health Association, National Conference on Tobacco or Health and TRDRP/CDPH joint scientific meetings.

\section{Discussion}

This project will provide scientific evidence of the consequences of removing filters from cigarettes. This is critical information needed to advance state or local legislation to ban the sales of filtered cigarettes. Such legislation would reduce the environmental impact of non-biodegradable cigarette butt remnants, although there might still be contamination from tobacco and paper butt remnants. The main health impact of this product ban would likely be the reduction of cigarettes smoked per day, the inducement of many smokers to quit smoking, and the discouragement of new smokers from starting. These outcomes could then be advocated in other states besides California and perhaps even internationally. These results may have a substantial impact in tobacco product use globally.

\section{Trial Status}

The trial is currently (February 2020) in the midst of subject enrollment.

\section{Abbreviations}

CO: carbon monoxide

CPD: Cigarettes per Day

DSMB: Data Safety and Monitoring Board

NNAL: urinary cotinine, 4-(methylnitrosamino)-1-(3-pyridyl)-1-butanol)

VOC: volatile organic compound

\section{Declarations}

\section{Acknowledgements}

This study would not have been possible without grant support from the American Legacy Foundation and Tobacco-related Disease Research Program. We are also grateful to study team members who have participated in the set-up and management of the study at San Diego State University and California State University, San Marcos.

\section{Authors' contributions $\{31 \mathrm{~b}\}$}


TEN and KP conceived the original study design. EO and KP updated and developed the study design with contributions from TEN and DRR. CB and EC created study forms, protocols and tools with support from EO and KP. KP drafted the manuscript and subsequent versions with substantial contributions from $\mathrm{EO}$ and TEN. All authors read and approved the final manuscript.

\section{Funding $\{4\}$}

This project is funded as a high impact pilot grant through the Tobacco-Related Disease Research Program.

\section{Availability of data and materials $\{29\}$}

The datasets used and/or analyzed during the current study are available from the corresponding author on reasonable request.

\section{Ethics approval and consent to participate $\{24\}$}

Approval Number: HS-2018-0152

Board Name: Human Research Protection Program (HRPP)

Board Affiliation: San Diego State University

\section{Consent for publication $\{32\}$}

Not applicable.

\section{Competing interests $\{28\}$}

The authors declare that they have no competing interests.

\section{References}

1. Novotny TE, Slaughter E. Tobacco product waste: An environmental approach to reduce tobacco consumption. Current Environmental Health Reports. 2014.

2. Slaughter E, Gersberg RM, Watanabe K, Rudolph J, Stransky C, Novotny TE. Toxicity of cigarette butts, and their chemical components, to marine and freshwater fish. Tobacco Control. 2011;20(Suppl 1):i25i9.

3. Healton CG, Cummings KM, O'Connor RJ, Novotny TE. Butt really? The environmental impact of cigarettes. Tobacco control. 2011;20(Suppl 1):i1-i.

4. Novotny TE, Hardin SN, Hovda LR, Novotny DJ, McLean MK, Khan S. Tobacco and cigarette butt consumption in humans and animals. Tobacco control. 2011;20(Suppl 1):i17-i20.

5. Harris B. The intractable cigarette 'filter problem'. Tobacco control. 2011;20(Suppl 1):i10-i6.

6. U.S. National Cancer Institute. Tobacco ControlMonograph No. 13. Bethesda, MD: US Department of Health and Human Services, Public Health Service, National Institutes of Health, National Cancer 
Institute; 2001.

7. National Cancer Institute. Risks Associated with Smoking Cigarettes with Low Machine-Measured Yields of Tar and Nicotine. Monograph 13. Bethesda, MD: Department of Health and Human Services, National Institutes of Health, National Cancer Institutes; October 2001.

8. Brooks DR, Austin JH, Heelan RT, Ginsberg MS, Shin V, Olson SH, et al. Influence of type of cigarette on peripheral versus central lung cancer. Cancer Epidemiology Biomarkers \& Prevention. 2005;14(3):57681.

9. Thun MJ, Carter BD, Feskanich D, Freedman ND, Prentice R, Lopez AD, et al. 50-year trends in smokingrelated mortality in the United States. New England Journal of Medicine. 2013;368(4):351-64.

10. California Legislature 2013-2014 Regular Session. Assembly Bill No. 1504, Introduced by Assembly Member Stone. http://leginfo.legislature.ca.gov/faces/billNavClient.xhtml?bill_id=201320140AB1504. January 14, 2014.

11. Tobacco products: single-use and multiuse components (2020).

12. Javors MA, Hatch JP, Lamb RJ. Cut-off levels for breath carbon monoxide as a marker for cigarette smoking. Addiction. 2005;100(2):159-67.

13. Cappelleri JC, Bushmakin AG, Baker CL, Merikle E, Olufade AO, Gilbert DG. Confirmatory factor analyses and reliability of the modified cigarette evaluation questionnaire. Addictive behaviors. 2007;32(5):91223.

14. California Department of Health. California Adult Tobacco Survey-2008 Questionnaire. http://www.cdph.ca.gov/programs/tobacco/Documents/CTCPCaliforniaAdultTobaccoSurvey2008.pdf; 2007.

15. Heatherton TF, Kozlowski LT, Frecker RC, Fagerstrom K-O. The Fagerstom Test for Nicotine Dependence: A revision of the Fagerstrom Tolerance Questionnaire. British Journal of Addiction. 1991;86(9):111927.

16. Etter J-Fo, Le Houezec J, Perneger TV. A self-administered questionnaire to measure dependence on cigarettes: The cigarette dependence scale. Neuropsychopharmacology. 2003;28(2):359-70.

17. Smith SS, Piper ME, Bolt DM, Fiore MC, Wetter DW, Cinciripini PM, et al. Development of the Brief Wisconsin Inventory of Smoking Dependence Motives. Nicotine Tob Res. 2010;12(5):489-99.

18. Clark PI, Gautam SP, Hlaing WM, Gerson LW. Response error in self-reported current smoking frequency by black and white established smokers. Annals of epidemiology. 1996;6(6):483-9.

19. Lee EM, Malson JL, Waters AJ, Moolchan ET, Pickworth WB. Smoking topography: reliability and validity in dependent smokers. Nicotine Tob Res. 2003;5(5):673-9.

20. Blank MD, Disharoon S, Eissenberg T. Comparison of methods for measurement of smoking behavior: mouthpiece-based computerized devices versus direct observation. Nicotine \& Tobacco Research. 2009;11(7):896-903.

21. Ashley DL, O'Connor RJ, Bernert JT, Watson CH, Polzin GM, Jain RB, et al. Effect of differing levels of tobacco-specific nitrosamines in cigarette smoke on the levels of biomarkers in smokers. Cancer Epidemiology Biomarkers \& Prevention. 2010;19(6):1389-98. 
22. Jacob III P, Havel C, Lee D-H, Yu L, Eisner MD, Benowitz NL. Subpicogram per milliliter determination of the tobacco-specific carcinogen metabolite 4-(methylnitrosamino)-1-(3-pyridyl)-1-butanol in human urine using liquid chromatography- tandem mass spectrometry. Analytical chemistry. 2008;80(21):8115-21.

23. Jacob III P, Yu L, Duan M, Ramos L, Yturralde O, Benowitz NL. Determination of the nicotine metabolites cotinine and $<i>$ trans $</ i>-3^{\prime}$-hydroxycotinine in biologic fluids of smokers and non-smokers using liquid chromatography-tandem mass spectrometry: Biomarkers for tobacco smoke exposure and for phenotyping cytochrome P450 2A6 activity. Journal of Chromatography B. 2011;879(3):267-76.

24. Benowitz NL, Hall SM, Stewart S, Wilson M, Dempsey D, Jacob P. Nicotine and carcinogen exposure with smoking of progressively reduced nicotine content cigarette. Cancer Epidemiology Biomarkers \& Prevention. 2007;16(11):2479-85.

25. Pulvers $K$, Hood A, Limas EF, Thomas MD. Female smokers show lower pain tolerance in a physical distress task. Addictive Behaviors. 2012;37(10):1167-70.

26. Limas EF. The effects of a social norm intervention on smokers' physical distress tolerance and smoking behavior: California State University San Marcos; 2014.

27. Mueller-Cohrs J. Analysis of Incomplete two-period cross-over trials with SAS PROC MIXED. . 2006.

\section{Tables}

Table 1. Study Measures and Timepoints

\begin{tabular}{|c|c|c|c|c|c|c|c|c|c|}
\hline & & & $\begin{aligned} 1 \\
\text { swi }\end{aligned}$ & & & & & & \\
\hline List of Measures & $\begin{array}{c}\text { Visit } \\
1\end{array}$ & 2 & 3 & 4 & Washout & 5 & 6 & 7 & 8 \\
\hline Questionnaires & & & & & All smoke filtered & & & & \\
\hline Demographics & $\mathrm{x}$ & & & & Pall Mall or Camels & & & & \\
\hline Smoking behavior, intention to quit & $\mathrm{x}$ & $\mathrm{x}$ & $\mathrm{x}$ & $\mathrm{x}$ & & $\mathrm{x}$ & $\mathrm{x}$ & $\mathrm{x}$ & $\mathrm{x}$ \\
\hline Knowledge and risk perceptions of filtered cigarettes & $\mathrm{x}$ & $\mathrm{x}$ & $\mathrm{x}$ & $\mathrm{x}$ & & $\mathrm{x}$ & $\mathrm{x}$ & $\mathrm{x}$ & $\mathrm{x}$ \\
\hline Fagerström Test for Nicotine Dependence & $\mathrm{x}$ & $\mathrm{x}$ & $\mathrm{x}$ & $\mathrm{x}$ & & $\mathrm{x}$ & $\mathrm{x}$ & $\mathrm{x}$ & $\mathrm{x}$ \\
\hline ATS Respiratory Symptoms & $\mathrm{x}$ & $\mathrm{x}$ & $\mathrm{x}$ & $\mathrm{x}$ & & $\mathrm{x}$ & $\mathrm{x}$ & $\mathrm{x}$ & $\mathrm{x}$ \\
\hline Modified Cigarette Evaluation Questionnaire & $\mathrm{x}$ & $\mathrm{x}$ & $\mathrm{x}$ & $\mathrm{x}$ & & $\mathrm{x}$ & $\mathrm{x}$ & $\mathrm{x}$ & $\mathrm{x}$ \\
\hline $\begin{array}{l}\text { Brief Wisconsin Inventory of Smoking Dependence } \\
\text { Motives }\end{array}$ & $\mathrm{x}$ & & & & & & & & $\mathrm{x}$ \\
\hline Height & $\mathrm{x}$ & & & & & & & & \\
\hline Weight & $\mathrm{x}$ & $\mathrm{x}$ & $\mathrm{x}$ & $\mathrm{x}$ & & $\mathrm{x}$ & $\mathrm{x}$ & $\mathrm{x}$ & $\mathrm{x}$ \\
\hline Blood pressure & $\mathrm{x}$ & $\mathrm{x}$ & $\mathrm{x}$ & $\mathrm{x}$ & & $\mathrm{x}$ & $\mathrm{x}$ & $\mathrm{x}$ & $\mathrm{x}$ \\
\hline Carbon monoxide & $\mathrm{x}$ & $\mathrm{x}$ & $\mathrm{x}$ & $\mathrm{x}$ & & $\mathrm{x}$ & $\mathrm{x}$ & $\mathrm{x}$ & $\mathrm{x}$ \\
\hline Urinary cotinine and creatinine sample for analysis & $\mathrm{x}$ & & & $\mathrm{x}$ & & $\mathrm{x}$ & & & $\mathrm{x}$ \\
\hline Urinary NNAL and VOC sample for analysis & $\mathrm{x}$ & & & $\mathrm{x}$ & & $\mathrm{x}$ & & & $\mathrm{x}$ \\
\hline $\begin{array}{l}\text { Smoking topography (measured on } 5 \text { days at home } \\
\text { and downloaded at the following visit) }\end{array}$ & & $\mathrm{x}$ & $\mathrm{x}$ & $\mathrm{x}$ & & & $\mathrm{x}$ & $\mathrm{x}$ & $\mathrm{x}$ \\
\hline $\begin{array}{l}\text { Cigarette butt count (collected at home and } \\
\text { returned at the following visit) }\end{array}$ & & $\mathrm{x}$ & $\mathrm{x}$ & $\mathrm{x}$ & & & $\mathrm{x}$ & $\mathrm{x}$ & $\mathrm{x}$ \\
\hline
\end{tabular}


Table 2. Participant Reimbursement Schedule 


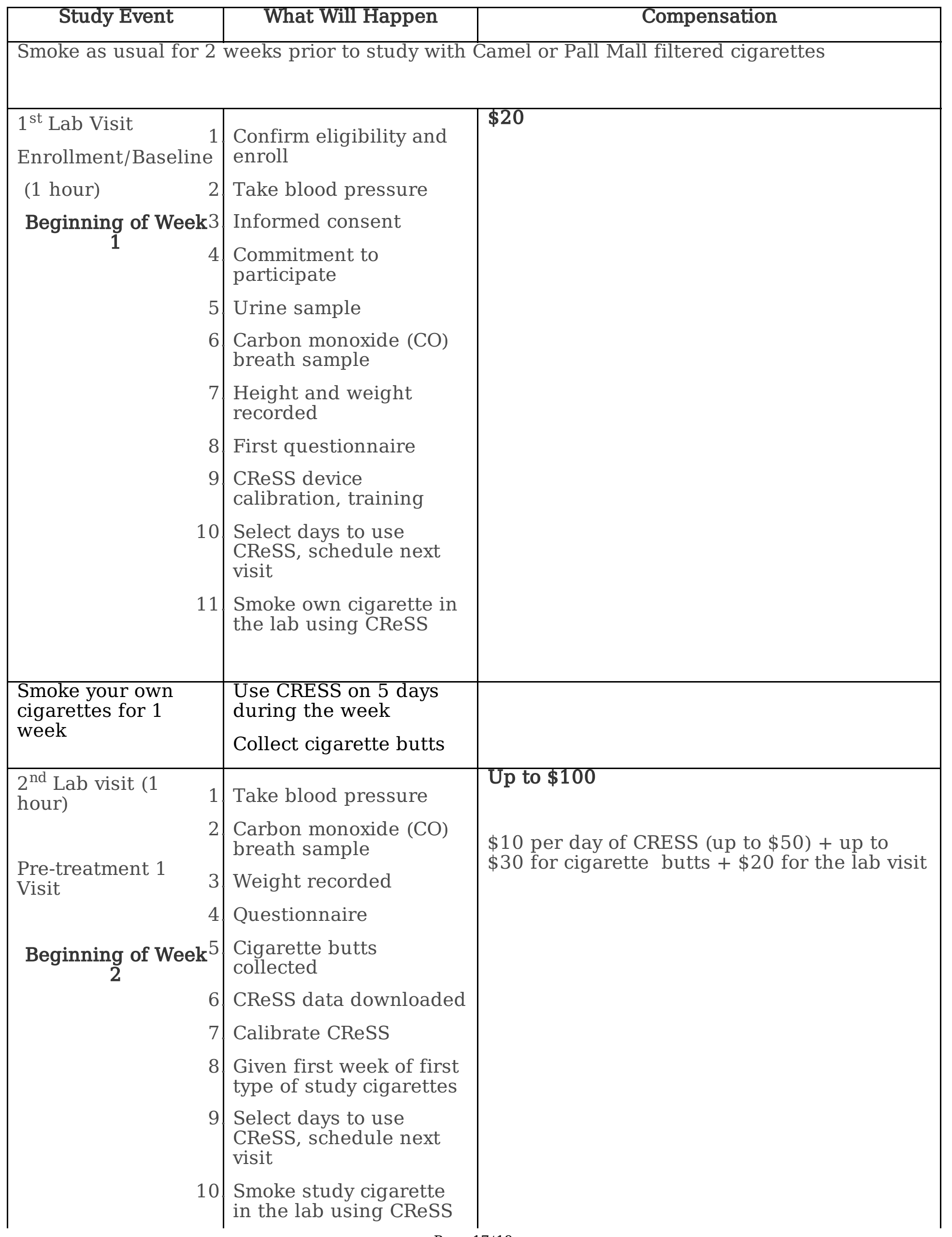


\begin{tabular}{|l|l|} 
& \\
\hline
\end{tabular}

CRESS Pocket: Handheld machine that you place your cigarette in while smoking; records "topography" such as puffing. We will send you home with the CRESS pocket so we can measure your smoking in your own environment as opposed to the lab environment. There are six weeks in which you will smoke using the CRESS Pocket, for an eight-hour period over five days. We will download your CRESS data at your lab visit and you will receive $\$ 10$ per day that you do this, for up to $\$ 50$ at a visit. We will determine whether the nicotine in your system matches the cigarettes per day that you report and your usage of the CRESS machine. Your CRESS incentive will be reduced if it appears that you are not using the machine as instructed. We will estimate a percentage of compliance and pay that amount. For example, if someone is $100 \%$ compliant they will receive full CRESS incentive. If someone is $50 \%$ compliant, they will receive $50 \%$ CRESS incentive. You are welcome to use the CRESS on more than five days and for longer than eight hours - we are requiring and incentivizing five days of eight-hour periods.

Carbon Monoxide (CO): Handheld Breathalyzer that you exhale into. It will provide an estimate of carbon monoxide in your lungs.

Urine: You will provide a sample ( $40 \mathrm{ml}$ minimum) in a cup during your lab visit. We will analyze your urine for cotinine and chemicals from cigarettes only.

Weight/Height: You will step on a scale without shoes. Your height will be measured once on the scale.

Cigarette Butt Count: You will receive two separate two-week supplies of study cigarettes: one set will be filtered, and one set will be unfiltered. You will keep the butts from these cigarettes in a sandwich-sized Ziploc bag or glass jar to be brought to each of your weekly lab visits during the active four-week study period. You will receive $\$ 30$ for bringing your used cigarette butts when they are due. Your butt incentive will be reduced if it appears that you are not collecting your butts as instructed.

\section{Figures}




\section{A RANDOMIZED CROSS-OVER CLINICAL TRIAL OF UNFILTERED CIGARETTES \\ Study Design}

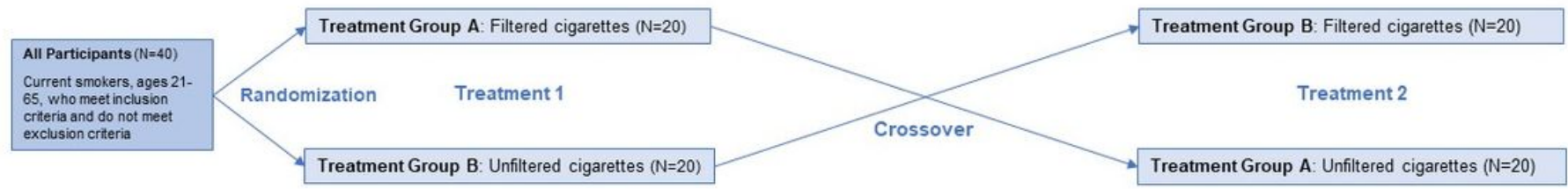

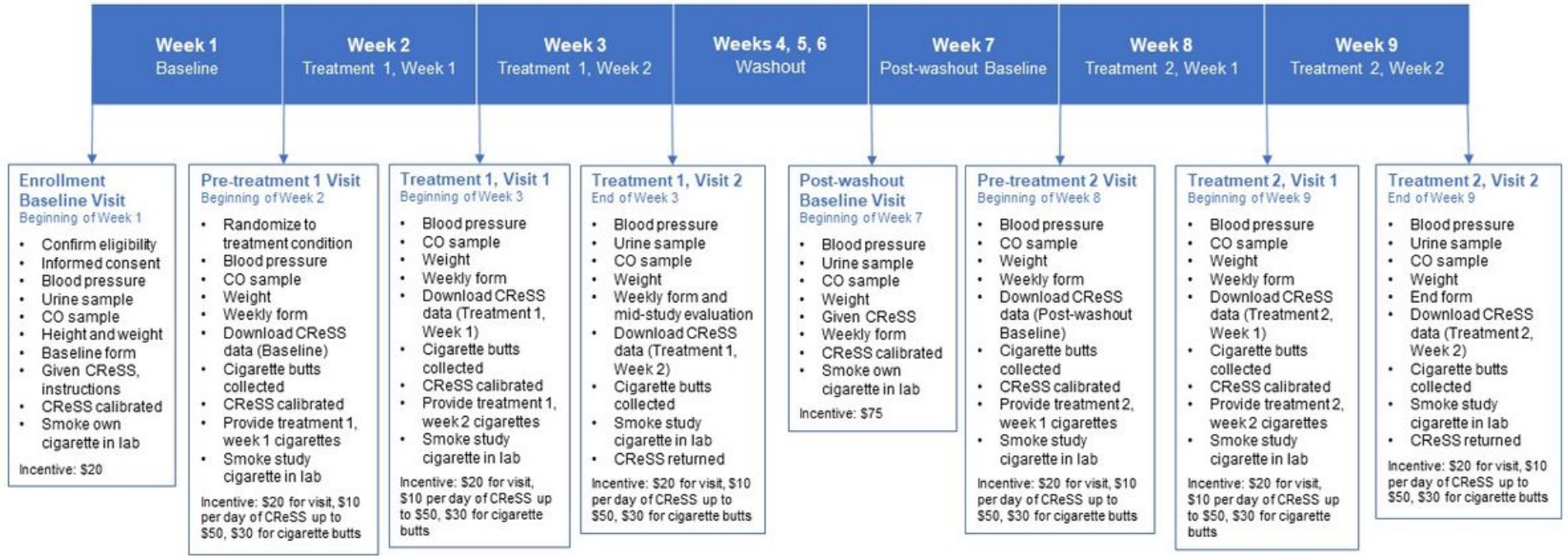

Figure 1

Study design diagram 